\title{
Applications of Nanomaterials and Nanotechnology in Oil and Gas Industry
}

\author{
Abdul Nasir Jawahar* \\ Lecturer in Department of Oil and Gas Engineering of Geology and Mines, Faculty in Jawzjan University, \\ Sheberghan, Afghanistan
}

*Corresponding Author: Abdul Nasir Jawahar, Lecturer in Department of Oil and Gas Engineering of Geology and Mines, Faculty in Jawzjan University, Sheberghan, Afghanistan.

\begin{abstract}
Nanotechnology has the potential to change the technology landscape of the oil and gas industry. Current research has already explored the applications of nanotechnology in vital sectors in the oil and gas industry from upstream exploration to downstream refining. This review had examined and evaluated the research progress in drilling and completion, enhanced/improved oil recovery using nanotechnology. Such information may help other researchers to understand the current research developments and challenges. Breakthroughs in fundamental research, including discovery of new synthesis method of nanomaterials, expansion of applications of existing nanomaterials, and explanations for the unique nanomaterials properties will continue facilitate the application of nanomaterials in the oil and gas industry.
\end{abstract}

Keywords: Nanomaterials, nanotechnology, oil industry, gas industry.

\section{INTRODUCTION}

Over the next 30 years, global energy demand is projected to rise as high as almost $60 \%$, a challenging trend that may be met only by revolutionary breakthroughs in energy science and technology. The industry needs stunning discoveries in underlying core science and engineering. Breakthroughs in nanotechnology open up the possibility of moving beyond the current alternatives for energy supply by introducing technologies that are more efficient and environmentally sound. Nanotechnology is characterized by collaboration among diverse disciplines, making it inherently innovative and more precise than other technologies. Such a technology may be the cornerstone of any future energy technology that offers the greatest potential for innovative solutions.

The laws of classical dynamics begin to deteriorate at high velocities and small scales where Einstein's relativity and quantum mechanics take over, respectively. Accordingly, small particles traveling at high velocities are subject to the laws of relativistic-quantum mechanics. Amazing phenomenon begin to occur in this domain, and new technology is trying to take advantage of these phenomenon to create miraculous machines. Quantum Mechanics becomes extremely important at these atomic scales.

The emergence of nanotechnology as a force in multidisciplinary research has led to the development of nanomaterials, where the oil and gas industry can adopt the principles of nanotechnology to solve practical problems encountered in oilfield development, especially in improving recovery efficiency of the oilfield. Several studies and reviews have documented the theory to practice in enhanced oil recovery where nanotechnology has been applied (Fletcher and Davis, 2010; Fathollahi and Rostami, 2015; Ayatollahi and Zerafat, 2012; ShamsiJazeyi et al., 2014). The impact of nanochemistry, including the use of polymer-coated nano particles, on the processes and mechanisms of enhanced oil recovery has been examined.

\section{IMPORTANCE OF NANOTECHNOLOGY IN INDUSTRY}

"Nano" denotes a thousand millionths (109), with a nanometer equaling a millionth of a millimeter. That corresponds to the width of 10 hydrogen atoms. A nanometer is merely ten angstroms long where an angstrom is named after Swedish astronomer and physicist, Ångström, Anders Jonas (18141874), who was one of the early formulators of the science of modern spectroscopy. Ångström wrote 
extensively on terrestrial magnetism, the conduction of heat, and especially spectroscopy. $\mathrm{He}$ published a monumental map of the normal solar spectrum that expressed the length of light waves in units of one ten-millionth of a millimeter. This unit of length usually used to specify radiation wavelengths is now known as the angstrom (10-10 meters). He discovered that hydrogen is present in the sun's atmosphere, and he was the first to examine the spectrum of the aurora borealis. Although, the diameter of atoms varies and the basic unit is taken to be the Bohr radius $\left(5.2917725 \times 10^{-11} \mathrm{~m}\right)$, the diameter of an atom depending upon the element is approximately one to five angstroms. This puts from 2 to 10 atoms in a nanometer and 2000 to 10000 atoms in a micrometer. For example, there are about 3 silicon atom diameters in a nanometer.

Nanotechnologist is concerned with building new structures and substances by manipulating molecules and atoms on this scale. Technically, nanotechnology is the art and science of building materials that act at the nanometer scale. It builds at the ultimate level of finesse, one atom at a time, and it does it with molecular perfection. In a general sense, nanotechnology is the ability to create and manipulate matter at the molecular level that makes it possible to create materials with improved (or, more accurately, altered) properties, such as being both lightweight and having ultrahigh strength, and greater capabilities such as in electrical and heat conductivity. Another research approach is known as top-down nanofabrication, which involves working with bulk materials and reducing them to nanometer size. This is most common in currently used technology development schemes.

Nanotechnology is poised to impact dramatically on all sectors of industry. In oil and gas applications, nanotechnology could be used to increase opportunities to develop geothermal resources by enhancing thermal conductivity, improving down hole separation, and aiding in the development of noncorrosive materials that could be used for geothermal-energy production. Nanoscale metals already have been used to delineate ore deposits for geochemical exploration. Nanotechnology can be used to improve the drilling process. Some specialized petroleum laboratory has developed an advanced fluid mixed with nano-sized particles and superfine powder that significantly improve drilling speed. This blend eliminates damage to the reservoir rock in the well, making it possible to extract more oil.

\subsection{Nanotechnology and Gas Industry}

Nanotechnology could be used to enhance the possibilities of developing unconventional and stranded gas resources. Near-term challenges focus on liquefied-natural-gas (LNG) infrastructure and efficiency, LNG quality, and developing gas-toliquids (GTL) technology. Midterm challenges include developing super pipelines; constructing floating GTL platforms; production, re-gasification, and storage issues; and compressed-natural-gas transport. Long-term issues to be addressed are production of methane hydrates and gas by wire- producing electricity at the location of the gas source and carrying the electricity by wire to market rather than the gas to market by pipeline. Nanotechnology can address the problems associated with accessing stranded natural-gas resources by developing nano-catalysts and nanoscale membranes for GTL production and creating nano-structured materials for compressed-natural gas transport or long-distance electricity transmission.

\subsection{Nanotechnology and Oil Industry}

The oil industry needs strong, stable materials in virtually all of its processes. By building up such substances on a nano-scale, it could produce equipment that is lighter, more resistant, and stronger. GP Nano Technology Group Ltd. in Hong Kong was one of the first to develop silicon carbide, a ceramic powder, in nano size. It yields exceptionally hard materials. The company is now investigating other composites and believes that nano-crystalline substances can contribute to harder, more wear-resistant and more durable drilling equipment. Nano-tubes have many potential applications within the oil industry. For instance, nano-tubes could be used to create lighter, stronger, and more corrosion-resistant structural materials in platforms for offshore drilling. Nanotechnology could help improve oil and gas production by making it easier to separate oil and gas in the reservoir - for instance, through improved understanding of processes at the molecular level. There are many other potential clean energy sources that could be enhanced through the use of nanotechnology. The practical application of nanotechnology in the oil sector is, fortunately, less frightening. It opens interesting prospects for improved oil recovery, not least through better understanding of processes at the interface between liquids and solids. The aim is to understand how oil and water can be separated more effectively. Nanotechnology could be applied to improved oil 
recovery in the form of tailoring surfactants. These can then be added to the reservoir in a more controlled way than with existing substances, thereby releasing more oil. It could also help develop new metering techniques with tiny sensors to provide improved information about the reservoir.

Nanomaterials play a multifunctional role in the drilling and completion process, especially in complex formations and extreme environments, such as high-temperature deep wells. There exist several advantages by adding nanomaterials into drilling and completion fluids (Alimohammadi et al., 2013; Sharma et al., 2012; Parizad and Shahbazi, 2016; Riley et al., 2012; William et al., 2014; Ismail et al., 2014)

- Stabilizing wellbore. Nanoparticles can form a compact filter cake on the wellbore, which can avoid or reduce the phenomena of water swelling and wellbore collapse.

- Reducing filter loss. By introducing absorbing polymers in drilling fluid, nanoparticles can seal off nano/micro-sized pores on the wellbore, reducing the loss of drilling fluid.

- Improving rheology. Nanoparticles can increase the apparent viscosity and dynamic shearing force of the drilling system, thereby improving rheological properties.

- Increasing thermal stability. Drilling and completion fluids that contain nanomaterials may possess higher temperature tolerance, and can be applied in drilling deep wells.

\subsection{Nanotechnology and Environment}

Pollution by chemicals or gases is a difficult aspect of petroleum production, but the signs are that nanotechnology can make the industry considerably greener. Filters and particles are now being developed with a nanostructure that allows them to remove volatile organic compounds from oil vapor and mercury from soil and water. Filters and membranes designed with nano-scale precision provide full control over what flow through. A new type of fluids, which can be labeled as "smart fluids," is becoming increasingly available to the oil and gas industry. These nano-fluids are designed by adding nanofabricated particles to a fluid in order to enhance or improve some of its properties. Essentially, nano-scale particles are suspended in the liquid phase in low volumetric fractions. The liquid phase can be any liquid such as oil, water, or conventional fluid mixtures. The nano-particles used in the design of such fluids are preferably inorganic with properties of no dissolution or aggregation in the liquid environment. They can be designed to be compatible with reservoir fluids and are environmentally friendly. Recent experiments have shown some promising nano-fluids with amazing properties such as fluids with advanced drag reduction, binders for sand consolidation, gels, products for wettability alteration, and anticorrosive coatings.

\subsection{Nanotechnology and Exploration}

In high-temperature/high-pressure conditions, old electrical sensors and other measuring tools often are not reliable. But researchers currently are developing a set of reliable and economical sensors from optical fibers for measuring temperature and pressure, oil-flow rate, and acoustic waves in oil wells. These new sensors are small in size, work safely in the presence of electromagnetic fields, are able to work in high temperatures and pressures, and can be changed at a sensible cost without interfering in the procedure of oil exploration. Changing and displacing old sensors in oil wells is very costly. But this technology could, with its accurate and reliable measurements, make a great improvement in oil exploration. In the future, the industry may be using nano-scale sensors for probing properties deep in the reservoir, allowing us to unravel the complex nature of the rock/fluid interactions and their effects on multiphase flow and providing the ability to design a suitable exploitation plan for the asset. Another area of significant challenge lies in the upgrading of bitumen and heavy crude oil. Because of their high density and viscosity, it is difficult to handle and transport them to locations where they could be converted into valuable products. Significant resources and intense research activities have been devoted to develop processes and specifically designed catalysts for on-site field upgrading combined with hydrogen/methane production. These processes would incorporate a minimized and controlled carbon rejection, in conjunction with a catalytically enhanced hydrogen generation performed on the rejected carbon from the upgrading process. This central activity will be complemented with an effort to integrate the research for ultra-dispersed catalytic formulas for the insitu upgrading of bitumen as well as for hydrogen generation from coal/coke or petroleum pitch. The 
former requires research on specifically designed adsorbents and catalysts to be introduced into the reservoir porous media in nano-sized form. The latter requires extensive research on catalytic active phases and process setup as well as adopting different catalytic forms for effective contact with the gasifying materials. This research has the potential to generate significant technology to convert bitumen and heavy-oil reserves into products cost-effectively.

\subsection{Nano-material Application}

People have wanted to be rich for centuries. People have been trying to create gold ever since gold became the measure of wealth. People have also been trying to make diamonds because not only are they expensive and a display of aristocracy, but also rare, and quite useful in industrial applications such as cutting. Unlike gold, diamonds are constructed of carbon which is readily available at dirt cheap prices. If anyone could cheaply put carbon atoms together to form diamonds, he would become rich instantly. Some of the possible benefits of nano-materials are the outcome of miniaturization, while others are the result of change in the property of the material. As readily accessible reserves become depleted, the oil and gas industry faces increasing technical challenges, which lead to increased costs and limit the operating envelope of drilling and production technologies. This represents a significant market opportunity for nano-material-based solutions, which contend with corrosive impurities, high temperatures and pressures, shock loads, abrasion, and other hostile environmental conditions. However, very few nano-material-based products have yet to appear in the oil and gas technology basket. This can be attributed to a number of factors, including: 1- lack of innovation in the E\&P sector 2- barriers to entry and adoption 3- perceived cost and risk 4-lack of awareness.

To facilitate this, oil and gas companies should enter into partnerships with nano-material developers at an early stage. Both sides need to accept that some necessary investment risk must be taken to bring nano-materials into the market. There are numerous areas in which nanotechnology can contribute to more-efficient, less-expensive, and more-environmentally sound technologies than those that are readily available. Although the most significant contributions may be unglamorous applications such as better materials for exploration equipment or improved catalysis, nanotechnology is being proposed in numerous energy domains. Considering the substantial budgets for research dedicated to nanoresearch, much of this potential is likely to be realized in the coming decades.

\section{CONCLUSION}

Nanotechnology has the potential to change the technology landscape of the oil and gas industry. Current research has already explored the applications of nanotechnology in vital sectors in the oil and gas industry from upstream exploration to downstream refining. This review examined and evaluated the research progress in drilling and completion, enhanced/improved oil recovery, heavy oil recovery, water treatment, nanomembranes, catalytic refining, and anti-corrosion. Breakthroughs in fundamental research, including discovery of new synthesis method of nanomaterials, expansion of applications of existing nanomaterials, and explanations for the unique nanomaterials properties will continue facilitate the application of nanomaterials in the oil and gas industry.

\section{REFERENCES}

[1] Alimohammadi N, S. R. Shadizadeh, I. Kazeminezhad. 2013. Removal of cadmium from drilling fluid using nano-adsorbent. Fuel. 111: 505-509.

[2] Ayatollahi S, M. M. Zerafat. 2012. Nanotechnology-Assisted EOR Techniques: New Solutions to Old Challenges, PE International Oilfield Nanotechnology Conference and Exhibition, Noordwijk, 12-14.

[3] Fathollahi A, B. Rostami. 2015. Carbonated water injection: Effects of silica nanoparticles and operating pressure. The Canadian Journal of Chemical Engineering 93: 1949-1956.

[4] Fletcher, A., J. Davis. 2010. How EOR can be transformed by Nanotechnology. SPE Improved Oil Recovery Symposium, Tulsa, 24-28.

[5] Ismail AR, N. M. Rasid, M. Z. Jaafar, W. R. W. Sulaiman, N. A. Buang, Effect of nanomaterial on the rheology of drilling fluids. Journal of Applied Sciences, 14: 1192-1197.

[6] Parizad, A., Shahbazi, K. (2016). Experimental investigation of the effects of SnO2nanoparticles and KCl salt on a water base drilling fluid properties. The Canadian Journal of Chemical Engineering, 94(10), 1924-1938. 
[7] Riley M, S. Young, E. Stamatakis, Q. Guo, L. Ji, G. De Stefano, K. Price, J. Friedheim. 2012. Wellbore Stability in Unconventional Shales - The Design of a Nano-Particle Fluid, SPE Oil and Gas India Conference and Exhibition, Mumbai, 28-30.

[8] Scott, S., Crudden, C.M., and Jones, C.W. (eds.). 2003. Nano-structured Catalysts. Nanostructure Science and Technology Series. Springer, New York, 342.

[9] Shamsijazeyi H, C. A. Miller, M. S. Wong, J. M. Tour, R. Verduzco. 2014. Polymer-coated nanoparticles for enhanced oil recovery. Journal of Applied Polymer Science, 131: 4401.

[10] Sharma MM, R. Zhang, M. E. Chenevert, L. Ji, Q. Guo, J. Friedheim, 2012. A New Family of Nanoparticle Based Drilling Fluids. SPE Annual Technical Conference and Exhibition, San Antonio, 810 .

[11] Smalley, R.E. and Yakobsonb, B.I. 1998. The Future of the Fullerenes. Solid State Communications, 107(11): 597-606.

[12] Wang, X., Cheng, Z., Lu, Y., Xu, L., and Xie, X. 1997. Nano-scaled Metals in Earth gas and Mobile Forms of Metals in Overburden in Wide-Spaced Regional Exploration for Giant Deposits in Overburden Terrains. J. of Geochemical Exploration, 58: 63-72.

[13] Wasan, D.T., and Nikolov, A.D. 2003. Spreading of Nanofluids on Solids. Nature 423,156-159.

[14] William JKM, Ponmani S, Samuel R, Nagarajan R, Sangwai JS. 2014. Effect of CuO and ZnO nanofluids in xanthan gum on thermal, electrical and high-pressure rheology of water-based drilling fluids. Journal of Petroleum Science and Engineering. 117: 15-27

[15] Ying, J.Y., and Sun, T. 1997. Research Needs Assessment on Nano-structured Catalysts. J. Electroceramics, 1(3): 219-238.

[16] Zhou, W., Heiney, P.A., Fan, H., Smalley, R.E., and Fischer, J.E. 2005. Single-Walled Carbon Nanotube Templated Crystallization of $\mathrm{H}_{2} \mathrm{SO}_{4}$ : Direct Evidence for Protonation. J. American Chemical Soc., 127: $1640-1641$.

Citation: Abdul Nasir Jawahar, (2020). “Applications of Nanomaterials and Nanotechnology in Oil and Gas Industry”, International Journal of Petroleum and Petrochemical Engineering (IJPPE), 6(1), pp.28-32, DOI: http://dx.doi.org/10.20431/2454-7980.0601004

Copyright:@ 2020Authors. This is an open-access article distributed under the terms of the Creative Commons Attribution License, which permits unrestricted use, distribution, and reproduction in any medium, provided the original author and source are credited. 